\title{
INFLUÊNCIA DA GRANULOMETRIA, DO DIÂMETRO E DO COMPRIMENTO DE AMOSTRAS DE GRÃOS TRITURADOS DE SOJA NA DETERMINAÇÃO DE COEFICIENTES SIMULTÂNEOS DE TRANSFERÊNCIA ${ }^{1}$
}

\author{
Kil Jin PARK ${ }^{2, *}$, Ana Paula ITO², Juliana Tófano de Campos LEITE ${ }^{2}$
}

\section{RESUMO}

Este trabalho teve como principal objetivo verificar a influência da granulometria de grãos de soja triturados, assim como do diâmetro e do comprimento do corpo-de-prova na determinação de coeficientes simultâneos de transferência de calor e massa. Utilizando-se equipamentos de coluna fechada e de coluna aberta, foram testadas três granulometrias, dois comprimentos e três diâmetros diferentes para as amostras, totalizando dezoito condições experimentais. Nos experimentos com o equipamento de coluna fechada, a maior influência na avaliação da condutividade térmica foi devida aos valores de fluxo de calor obtidos. Tanto nos experimentos de coluna fechada quanto de coluna aberta, a contribuição dos fenômenos simultâneos deve ser considerada. O comprimento e a granulometria têm influência na determinação da condutividade térmica e na difusividade mássica, ao passo que o diâmetro só interfere na obtenção do valor da difusividade mássica.

Palavras-chave: condutividade térmica; difusividade mássica; gradiente de temperatura; gradiente de concentração de umidade.

\section{SUMMARY}

INFLUENCE OF SOYBEAN GRAIN SIZING AND SAMPLE DIAMETER AND LENGTH ON THE DETERMINATION OF SIMULTANEOUS TRANSFER RATES. The main goal of this work was to verify the influence of triturated soybean grain sizing, their sample diameters and lengths, on the determination of simultaneous heat and mass transfer rates. Using equipment with open and closed columns, three different grain sizings were tested, totalling eighteen different test conditions. For experiments with the closed column, the greater influence on the evaluation of the thermal conductivity was due to the heat flow rates. On experiments with the closed column as well as on those with the open column, the contribution of the simultaneous phenomena must be acknowledged. The sample length and the grain sizing can influence both heat conductivity and mass diffusivity, while the sample diameter interferes only with mass diffusivity rates.

Keywords: thermal conductivity; mass diffusivity; heat gradient; humidity concentration gradient.

\section{1 - INTRODUÇÃO}

No projeto de equipamentos e nos processos que envolvem transferência de calor, tais como: secagem, refrigeração, congelamento, evaporação e aquecimento, torna-se de grande importância o conhecimento das propriedades termofísicas dos materiais. Em especial, quando se deseja conhecer o tempo de duração de um dado processo, os coeficientes fenomenológicos do material precisam ser conhecidos.

Os métodos que podem ser usados na determinação experimental da condutividade térmica são classificados genericamente em regime permanente e regime transiente. No primeiro, os valores locais da temperatura são constantes em relação ao tempo e, no segundo, os valores locais da temperatura variam em relação ao tempo.

O método em regime permanente apresenta a desvantagem de requerer muito tempo para realizar o experimento, o que pode causar migração de umidade da amostra, principalmente para materiais biológicos, além de erros devido à resistência de contato entre a parte metálica e a amostra e perdas de calor ao ambiente.

Os coeficientes fenomenológicos cujo conhecimento é importante são: $\mathrm{K}_{\mathrm{t}}, \mathrm{K}_{\mathrm{m}}, \mathrm{D}_{\mathrm{m}}$ e $\mathrm{D}_{\mathrm{t}}$, ocorrendo em equações de transferência simultânea de massa e de calor, onde $\mathrm{K}_{\mathrm{t}}$ é a condutividade térmica, $\mathrm{D}_{\mathrm{m}}$ é a difusividade de massa, $\mathrm{D}_{\mathrm{t}}$ é o coeficiente de migração da umidade induzida pelo gradiente de temperatura (efeito Soret) e $\mathrm{K}_{\mathrm{m}}$ é o coeficiente de transferência de calor induzida pelo gradiente de umidade (efeito Dufour). A determinação desses coeficientes visa avaliar o efeito do gradiente de temperatura na migração de umidade em diferentes tipos de alimentos.

Uma forma de se determinar estes coeficientes é através dos equipamentos de coluna aberta e de coluna fechada, construídos por SHIKI \& PARK [4].

Neste trabalho, foram determinados os coeficientes $\mathrm{K}_{\mathrm{t},} \mathrm{K}_{\mathrm{m},} \mathrm{D}_{\mathrm{t}}$ e $\mathrm{D}_{\mathrm{m}}$ para grãos triturados de soja através da utilização dos equipamentos de coluna fechada e de coluna aberta.

Foram avaliadas três diferentes granulometrias com diferentes dimensões de corpos-de-prova, a fim de se determinar a influência dos mesmos sobre a condutividade térmica e a difusividade mássica do material. Para determinação da condutividade térmica, foram avaliados os fluxos de calor através da fonte quente e da fonte fria.

\section{2 - MATERIAL E MÉTODOS}

\section{1 - Obtenção de amostra e corpo-de-prova}

Os corpos-de-prova utilizados foram obtidos a partir de grãos triturados de soja, separados por classes de tamanho. Essa separação foi efetuada através de um

\footnotetext{
${ }^{1}$ Recebido para publicação em 30/04/01. Aceito para publicação em 20/11/01.

${ }^{2}$ FEAGRI/UNICAMP - DPPPAG, Cx. Postal.6011, CEP 13081-970, Campinas-SP, Fone-Fax: (OXX19) 3788-1076, e-mail: kil@agr.unicamp.br

* A quem a correspondência deve ser enviada.
} 
conjunto de peneiras, indicadas para a granulometria de grãos, com as seguintes aberturas: \#28 (0,590mm); \#48 (0,297mm) e \# 100 (0,149mm).

Os grãos triturados e separados por classe de tamanho foram colocados no interior dos cilindros que compõem diferentes dimensões dos corpos-de-prova.

\section{2 - Equipamentos de coluna aberta e de coluna fe- chada}

A transferência de massa tradicional é normalmente descrita pela lei de Fick [5]. A principal hipótese na formulação da transferência de massa é que a força motriz da migração da água do material é devida somente ao gradiente de umidade. LUIKOV \& MIKHAILOV [1] e PHILIP \& DE VRIES [3] propuseram sistemas de equações para transferências simultâneas de massa e calor em um meio poroso. Esses pesquisadores sugeriram que o gradiente de temperatura é mais uma força motriz para o fluxo de água em materiais porosos. As equações para transferência simultânea de calor e massa, considerando constantes os coeficientes, são descritas a seguir:

$$
\begin{aligned}
& \mathrm{qh}=\mathrm{K}_{\mathrm{t}} \frac{\Delta \mathrm{T}}{\Delta \mathrm{X}}+\mathrm{K}_{\mathrm{m}} \frac{\Delta \mathrm{M}}{\Delta \mathrm{X}} \\
& \mathrm{qm}=\mathrm{D}_{\mathrm{t}} \frac{\Delta \mathrm{T}}{\Delta \mathrm{X}}+\mathrm{D}_{\mathrm{m}} \frac{\Delta \mathrm{M}}{\Delta \mathrm{X}}
\end{aligned}
$$

Onde:

$\mathrm{qh}$ = fluxo de calor $\left(\mathrm{W} / \mathrm{m}^{2}\right)$;

$\mathrm{qm}=$ fluxo de massa $\left(\mathrm{m}^{3} / \mathrm{m}^{2} \mathrm{~s}\right)$;

$\mathrm{K}_{\mathrm{t}}=$ condutividade térmica $(\mathrm{W} / \mathrm{m} \mathrm{K})$;

$\mathrm{K}_{\mathrm{m}}=$ coeficiente do efeito Dufour $(\mathrm{W} / \mathrm{m})$;

$\mathrm{D}_{\mathrm{m}}=$ difusividade mássica $\left(\mathrm{m}^{2} / \mathrm{s}\right)$;

$\mathrm{D}_{\mathrm{t}}=\delta \mathrm{T} * \mathrm{D}_{\mathrm{M}}=$ coeficiente do efeito Soret $\left(\mathrm{m}^{2} / \mathrm{s} \mathrm{K}\right)$;

$\mathrm{T}=$ temperatura $(\mathrm{K})$;

$\mathrm{M}=$ umidade $\left(\mathrm{m}^{3} / \mathrm{m}^{3}\right)$;

$\mathrm{X}=$ direção do fluxo.

Normalmente, $\mathrm{D}_{\mathrm{t}}$ é escrito como razão de $\mathrm{D}_{\mathrm{m}}$ usando um fator $\delta_{t}$ [2]. Assim sendo, a determinação de $D_{t}$ é equivalente à determinação de $\delta_{t}$. De modo que:

$\delta_{t}=\frac{\mathrm{D}_{t}}{D_{m}}$

Os equipamentos de coluna aberta e de coluna fechada utilizados neste experimento estão apresentados nas Figuras 1 e 2 .

A Figura 1 apresenta um desenho esquemático do equipamento de coluna fechada, o qual consiste em um cubo de Teflon com um furo cilíndrico no centro, no interior do qual foi acondicionada a amostra.

A Figura 2 mostra um desenho esquemático do equipamento de coluna aberta, o qual consiste em um cubo de Teflon com um furo cilíndrico no centro, no interior do qual foi acondicionada a amostra. O cubo de Teflon com a amostra foram colocados no interior de uma caixa acrílica provida de sistema de controle de temperatura.

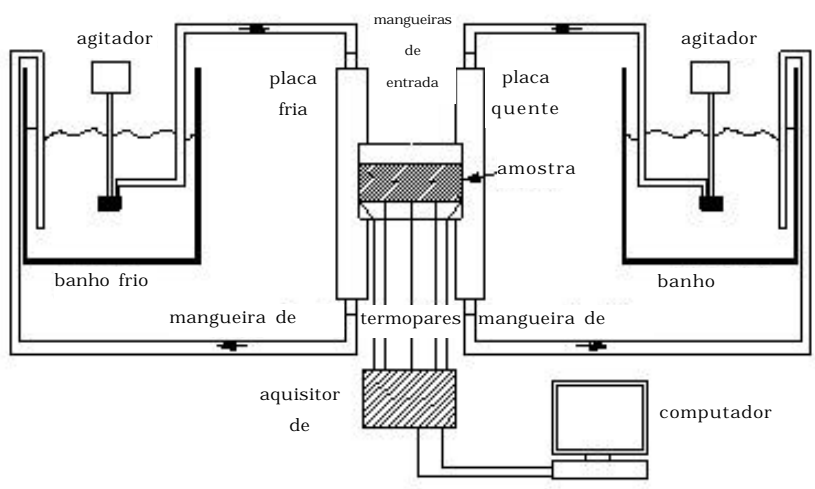

FIGURA 1. Esquema do equipamento de coluna fechada

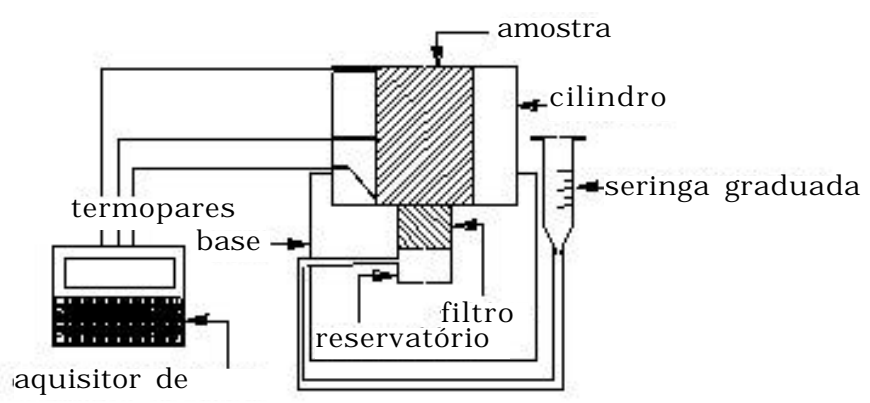

FIGURA 2. Esquema do equipamento de coluna aberta

Para que fosse possivel avaliar diferentes dimensões dos corpos-de-prova, diferentes cubos foram construídos com diferentes medidas de diâmetro (1, 2 e 3cm) e comprimento $(2,5$ e $5 \mathrm{~cm})$, para cada equipamento.

O princípio de funcionamento do equipamento de coluna fechada é baseado em um fluxo de calor constante e perda líquida de água nula. Obteve-se este fluxo de calor através da circulação de água quente e fria, a temperaturas constantes, no interior de placas de alumínio posicionadas nas extremidades opostas da amostra cilindrica. A amostra, no interior do cubo, foi colocada na posição horizontal (com o eixo longitudinal perpendicular ao eixo de ação gravitacional), com a finalidade de se evitar a migração de umidade induzida pela força da gravidade.

Através de orifícios igualmente espaçados no plano longitudinal do cubo de Teflon, foram inseridos termopares na amostra, os quais foram conectados a um equipamento de aquisição de dados capaz de armazenar medidas de temperatura realizadas em um intervalo de 60 segundos (valor especificado para o experimento). Para as amostras de $2,5 \mathrm{~cm}$ de comprimento, adotaram-se 3 pontos de medida de temperatura e, para as amostras de $5,0 \mathrm{~cm}$ de comprimento, 5 pontos de medida de temperatura.

O princípio de funcionamento do equipamento de coluna aberta é baseado no estabelecimento de um fluxo de massa através da amostra. 
Para que seja possível estabelecer esse fluxo, as amostras foram colocadas no interior do cubo de Teflon, de forma a garantir o isolamento térmico lateral. A extremidade superior da amostra ficou livre e em contato com o ar ambiente, de modo a permitir a evaporação de água. A extremidade inferior, por sua vez, foi colocada em contato com um filtro de papel, o qual foi posto em contato direto com um reservatório de água, também envolto por uma superfície isolante de Teflon (Figura 2). Dessa forma, a umidade relativa na extremidade inferior pode ser considerada igual a $100 \%$.

O reservatório de água foi conectado a uma seringa graduada por um pequeno orifício em sua base inferior, através da qual a água evaporada era reposta periodicamente, de forma a manter o nível constante. Através da medida da quantidade de água adicionada em certo período de tempo, foi possível determinar o fluxo de massa.

Para haver um melhor controle das condições ambientais no experimento de coluna aberta, o equipamento mostrado na Figura 2 foi colocado em uma caixa de acrílico dentro da qual havia circulação de ar com temperatura controlada. A circulação do ar foi realizada por um ventilador. Neste experimento, a temperatura utilizada foi de $30^{\circ} \mathrm{C}$.

Para determinar o gradiente de temperatura ao longo da amostra, termopares foram posicionados ao longo do seu eixo longitudinal, de maneira análoga ao equipamento de coluna fechada. O experimento foi conduzido durante 72 horas, sendo esse o tempo necessário para a amostra atingir a transferência de massa em regime permanente.

Ao final do experimento, as amostras foram divididas em três seções transversais equivalentes, pesadas e colocadas em estufa de convecção forçada por 24 horas a uma temperatura de $105^{\circ} \mathrm{C}$ para determinar a umidade em cada seção.

\section{3 - Cálculo dos coeficientes}

Utilizando os diferentes gradientes de temperatura experimentais e os diferentes dados de gradiente de umidade (na direção longitudinal " $x$ " da amostra) nas equações (1) e (2), todos os coeficientes combinados foram determinados. De acordo com a equação (3), o coeficiente $D_{t}$ pode ser representado pela relação $\delta_{t} D_{m}$.

Para a coluna fechada, "q" foi calculado com base na diferença de temperatura média ao longo das placas de alumínio situadas nas duas extremidades do cilindro, enquanto que $\mathrm{q}_{\mathrm{m}}=0$.

Para a coluna aberta, $\mathrm{q}_{\mathrm{m}}$ foi calculado com base no fluxo de água provocado pela evaporação, no estado estacionário, enquanto que $\mathrm{q}_{\mathrm{h}=0 \text {. }}$.

Dessa forma, tem-se um total de quatro equações para a determinação de quatro coeficientes.

Para determinação do fluxo de calor (q) através da amostra no equipamento de coluna fechada, considerou-se a diferença de temperatura ao longo da placa de alumínio, situada nas extremidades do cilindro. Os experimentos forneceram dois valores de q, sendo qh o fluxo obtido a partir da placa de alumínio que recebeu o banho quente e qc, pela placa que recebeu o banho frio. O valor de q foi calculado utilizando a equação (4):

$\mathrm{q}=\mathrm{k} \frac{\Delta \mathrm{T}}{\Delta \mathrm{x}}$

Onde:

$\mathrm{q}=$ quantidade de calor transferido entre a placa e a amostra $\left(\mathrm{W} / \mathrm{cm}^{2}\right)$;

$\mathrm{k}=$ condutividade térmica da liga de alumínio $2024=$ $1,22 \mathrm{~W} / \mathrm{cm} \mathrm{K}$

$\Delta \mathrm{T}=$ média da variação de temperatura entre as placas e amostra em K;

$\Delta \mathrm{x}=$ espessura da placa de alumínio entre a água e a amostra $=0,3 \mathrm{~cm}$.

\section{4 - RESULTADOS E DISCUSSÃO}

\section{1 - Equipamento de coluna fechada}

Utilizando-se a equação (4), calcularam-se os valores dos fluxos de calor $\mathrm{q}_{\mathrm{h}}$ e $\mathrm{q}_{\mathrm{c}}$ para cada amostra. A partir desses valores, calcularam-se os coeficientes $\mathrm{K}_{\mathrm{t}}$ e $\mathrm{K}_{\mathrm{m}}$ para cada condição experimental.

As Tabelas 1 e 2 apresentam valores de $\mathrm{K}_{\mathrm{t}}, \mathrm{K}_{\mathrm{m}}$ e $\mathrm{K}_{\mathrm{t}}^{\circ}$ (considerando $\mathrm{dM} / \mathrm{dX}=0$ ), para ambos os fluxos de calor, obtidos para os corpos-de-prova de 2,5 e $5,0 \mathrm{~cm}$ de comprimento, respectivamente, com diâmetros de 1,0 2,0 e $3,0 \mathrm{~cm}$ e granulometrias de peneiras 28 (abertura 0,590mm), 48 (abertura de $0,297 \mathrm{~mm}$ ) e 100 (abertura de $0,149 \mathrm{~mm})$.

TABELA 1. Valores de $\mathrm{K}_{\mathrm{t}}$ e $\mathrm{K}_{\mathrm{m}}$ obtidos para as amostras de $2,5 \mathrm{~cm}$ de comprimento, para diferentes condições de diâmetro e granulometria

\begin{tabular}{|c|c|c|c|c|c|c|c|}
\hline \multirow[t]{3}{*}{$\mathrm{D}(\mathrm{cm})$} & \multirow[t]{3}{*}{ Peneira } & \multicolumn{2}{|c|}{ Para qh } & \multicolumn{2}{|c|}{ Para qc } & \multicolumn{2}{|c|}{$\mathrm{dM} / \mathrm{dx}=0$} \\
\hline & & $\overline{\mathrm{K}_{\mathrm{t}}}$ & $\overline{\mathrm{m}}$ & $\overline{\mathrm{K}}$ & $\mathrm{K}_{\mathrm{m}}$ & Para qh $\mathrm{K}_{\mathrm{t}}^{0}$ & $\overline{\text { Para qc } \mathrm{K}_{\mathrm{t}}{ }^{0}}$ \\
\hline & & $(\mathrm{W} / \mathrm{m} \mathrm{K})$ & $(\mathrm{W} / \mathrm{m})$ & $\mathrm{W} / \mathrm{m} \mathrm{K})$ & $(\mathrm{W} / \mathrm{m})$ & (W/m K) & (W/m K) \\
\hline \multirow[t]{3}{*}{1} & \#28 & 0,0290 & $-0,4638$ & 0,0311 & $-0,4982$ & 0,0313 & 0,0158 \\
\hline & \#48 & 0,1314 & $-0,2003$ & 0,0705 & $-0,1075$ & 0,0306 & 0,0157 \\
\hline & \#100 & 0,0342 & $-0,2042$ & 0,0455 & $-0,2719$ & 0,0251 & 0,0127 \\
\hline \multirow[t]{3}{*}{2} & \#28 & 0,0218 & $-0,7964$ & 0,0436 & $-1,5966$ & 0,0274 & 0,0139 \\
\hline & \#48 & 0,0416 & $-0,3201$ & 0,0419 & $-0,3227$ & 0,0308 & 0,0156 \\
\hline & \#100 & 0,0090 & $-0,1242$ & 0,0253 & $-0,3489$ & 0,0237 & 0,0120 \\
\hline \multirow[t]{3}{*}{3} & \#28 & 0,0371 & $-0,2747$ & 0,0226 & $-0,1673$ & 0,0273 & 0,0138 \\
\hline & \#48 & 0,0459 & $-0,0965$ & 0,0463 & $-0,0973$ & 0,0286 & 0,0145 \\
\hline & \#100 & 1,8192 & $-0,0226$ & 1,7074 & $-0,0212$ & 0,0241 & 0,0122 \\
\hline
\end{tabular}

A partir das Figuras 3 e 4 é possivel avaliar a influência da granulometria na determinação da condutividade térmica, quando o gradiente mássico é considerado desprezivel. A Figura 3 representa a variação de $\mathrm{K}_{\mathrm{t}}^{\circ}$ com a granulometria para os corpos-de-prova de $2,5 \mathrm{~cm}$ de comprimento. A Figura 4 corresponde aos corpos-deprova de $5,0 \mathrm{~cm}$ de comprimento. 
TABELA 2. Valores de $\mathrm{K}_{\mathrm{t}}$ e $\mathrm{K}_{\mathrm{m}}$ obtidos para as amostras de $5,0 \mathrm{~cm}$ de comprimento, para diferentes condições de diâmetro e granulometria

\begin{tabular}{cccccccc}
\hline D (cm) & Peneira & \multicolumn{2}{c}{ Para qh } & \multicolumn{2}{c}{ Para qc } & \multicolumn{2}{c}{$\mathrm{dM} / \mathrm{dx}=0$} \\
\cline { 3 - 8 } & & $\begin{array}{c}\mathrm{K}_{\mathrm{t}} \\
(\mathrm{W} / \mathrm{m} \mathrm{K})\end{array}$ & $\begin{array}{c}\mathrm{K}_{\mathrm{m}} \\
(\mathrm{W} / \mathrm{m})\end{array}$ & $\begin{array}{c}\mathrm{K}_{\mathrm{t}} \\
\mathrm{W} / \mathrm{m} \mathrm{K})\end{array}$ & $\begin{array}{c}\mathrm{K}_{\mathrm{m}} \\
(\mathrm{W} / \mathrm{m})\end{array}$ & $\begin{array}{c}\text { Para qh } \mathrm{K}_{\mathrm{t}}{ }^{0} \\
(\mathrm{~W} / \mathrm{m} \mathrm{K})\end{array}$ & $\begin{array}{c}\text { Para } \\
(\mathrm{W} / \mathrm{m} \mathrm{K})\end{array}$ \\
\hline 1 & $\# 28$ & 0,0588 & $-2,8018$ & 0,0170 & $-0,8084$ & 0,0487 & 0,0247 \\
& $\# 48$ & 0,0828 & $-0,5411$ & 0,0003 & $-0,0020$ & 0,0548 & 0,0277 \\
& $\# 100$ & 0,0409 & $-0,4124$ & 0,0568 & $-0,5720$ & 0,0491 & 0,0248 \\
2 & $\# 28$ & 0,0646 & 0,3855 & $-0,0497$ & 0,2967 & 0,0508 & 0,0257 \\
& $\# 48$ & 0,0628 & $-1,2343$ & 0,0591 & $-1,1620$ & 0,0540 & 0,0273 \\
& $\# 100$ & 0,0600 & $-1,4231$ & 0,0434 & $-1,0292$ & 0,0528 & 0,0267 \\
& $\# 28$ & 0,1690 & $-1,1415$ & 0,1384 & $-0,9359$ & 0,0599 & 0,0303 \\
& $\# 48$ & 0,0521 & $-0,3250$ & 0,4488 & $-0,3220$ & 0,0494 & 0,0250 \\
& $\# 100$ & 0,0211 & $-0,9951$ & 0,0269 & $-1,2572$ & 0,0481 & 0,0243 \\
\hline
\end{tabular}

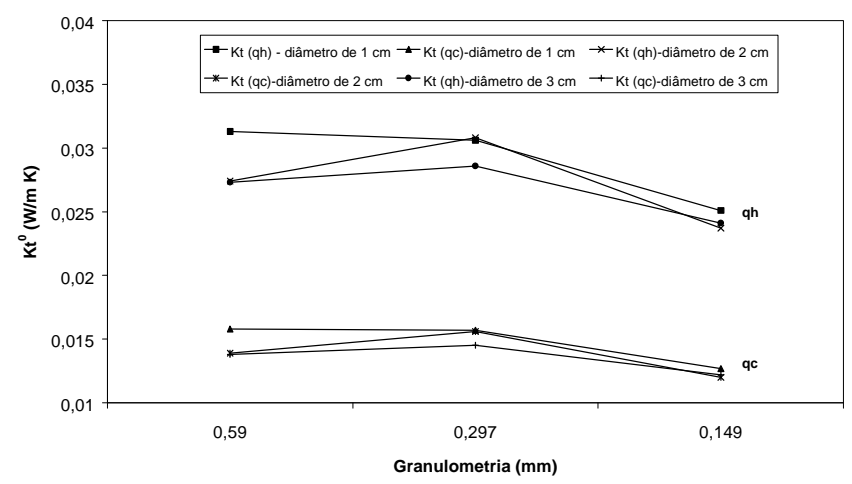

FIGURA 3. Influência da granulometria na determinação da condutividade térmica para as amostras de $2,5 \mathrm{~cm}$ de comprimento, considerando-se $\mathrm{dM} / \mathrm{dX}=0$.

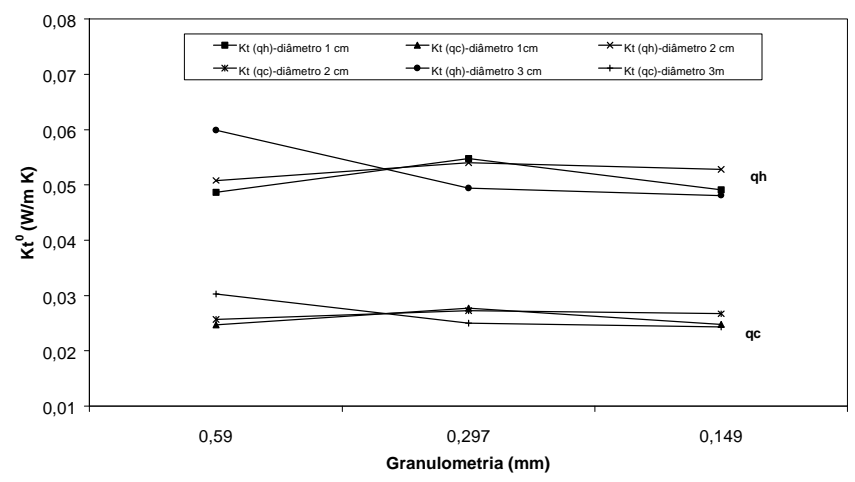

FIGURA 4. Influência da granulometria na determinação da condutividade térmica para as amostras de $5,0 \mathrm{~cm}$ de comprimento, considerando-se $\mathrm{dM} / \mathrm{dX}=0$.

A análise das Figuras 3 e 4 mostra que a granulometria não exerceu grande influência na condutividade térmica das amostras, quando o gradiente de umidade foi desconsiderado, havendo pequena variação dos valores em torno da média.

O que é possível perceber, pela análise das Figuras 3 $e 4$, é que os valores de $\mathrm{K}_{\mathrm{t}}^{\circ}$ são cerca de duas vezes maiores, considerando-se o mesmo ponto, para os fluxos de calor obtidos a partir da placa quente (qh), do que para os fluxos de calor obtidos a partir da placa fria (qc), de- monstrando a necessidade de melhor avaliar a quantidade de calor transferida na amostra.

A Figura 5 mostra a influência do comprimento dos corpos-de-prova na determinação da condutividade térmica quando o gradiente mássico é considerado desprezivel, para as três diferentes granulometrias (\#28, \#48 e \# 100) e para os três diâmetros (1,0; 2,0 e 3,0cm), considerando-se o fluxo de calor a partir da placa quente (qh). A Figura 6 mostra a mesma situação, considerando-se o gradiente mássico.

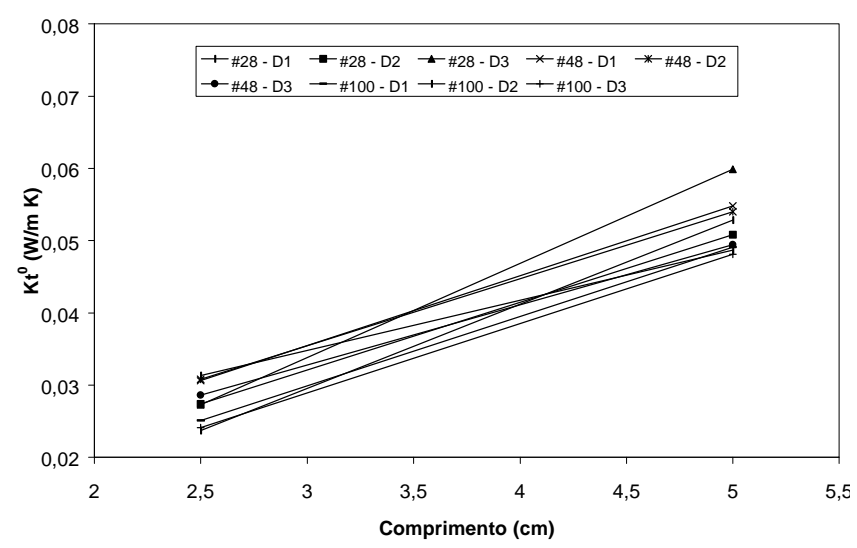

FIGURA 5. Influência do comprimento na determinação da condutividade térmica $\left(\mathrm{K}_{\mathrm{t}}^{\circ}\right)$ para $\mathrm{dM} / \mathrm{dX}=0$

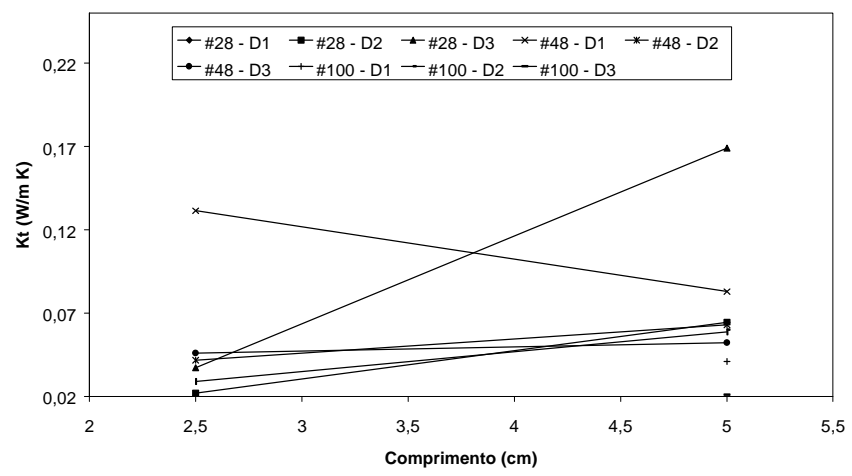

FIGURA 6. Influência do comprimento na determinação da condutividade térmica $\left(\mathrm{K}_{\mathrm{t}}\right)$ para $\mathrm{dM} / \mathrm{dX} \neq 0$

Pela Figura 5, onde o gradiente mássico não é considerado, observa-se que o comprimento tem influência na determinação de $\mathrm{K}_{\mathrm{t}}$. Percebe-se que ocorre um aumento da condutividade com o aumento do comprimento do corpo-de-prova, para todas as amostras.

Porém, se comparadas as Figuras 5 e 6, percebe-se que as linhas de variação da condutividade térmica com o comprimento não apresentam a mesma tendência quando o gradiente de umidade é considerado. Isso evidencia que o gradiente mássico não pode ser considerado desprezivel, mesmo na coluna fechada.

Analisando-se a Figura 6, percebe-se que, mesmo considerando-se a existência do gradiente mássico, continua a existir variação da condutividade térmica do 
material com o comprimento. Porém, a determinação da umidade da amostra envolve várias fontes de erro, tais como a concentração de umidade na superfície da placa fria, devido à condensação de vapor d'água presente no ar; a imprecisão no corte da amostra em seções equivalentes e o tempo decorrido entre a retirada da amostra do cubo de Teflon e a sua pesagem para determinação de umidade, permitindo a migração de umidade da amostra. Desta forma, pode-se concluir que a influência do comprimento da amostra na determinação da condutividade é resultante da contribuição de uma migração de umidade.

A Figura 7 mostra a influência da variação do diâmetro da amostra na determinação da condutividade térmica, para as amostras com 2,5 e $5,0 \mathrm{~cm}$ de comprimento, para cada uma das granulometrias (\#28, \#48 e \#100), considerando-se o gradiente mássico desprezível e o fluxo de calor a partir da placa quente.

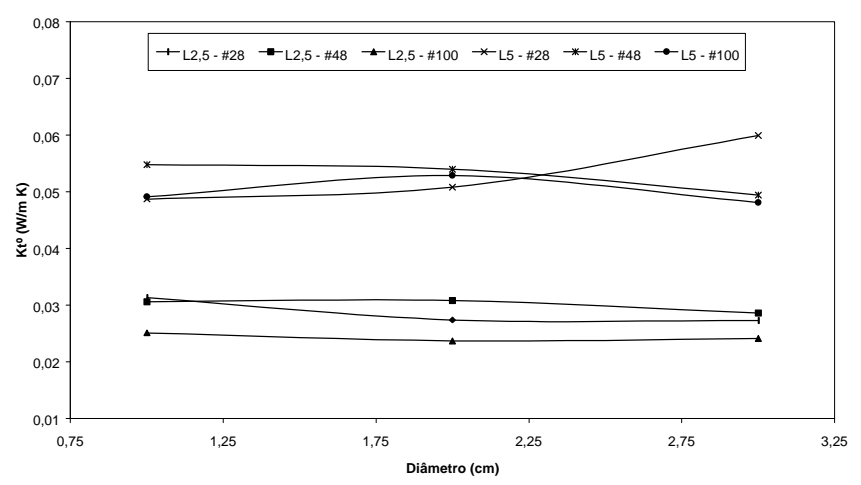

FIGURA 7. Influência do diâmetro da amostra na determinação da condutividade térmica, para $d M / d X=0$

Pela observação da Figura 7, pode-se concluir que a influência do diâmetro da amostra na determinação da condutividade térmica pode ser considerada desprezível, para todas as condições experimentais, isto é, a transferência de calor na direção radial é desprezivel.

\section{2 - Equipamento de coluna aberta}

No equipamento de coluna aberta, foram obtidos dados de temperatura e umidade ao longo de cada uma das amostras, de modo que foi possivel estabelecer os gradientes de temperatura e umidade $(\Delta \mathrm{T} /$ $\Delta \mathrm{X}$ e $\Delta \mathrm{M} / \Delta \mathrm{X}$, respectivamente) para cada condição experimental. O valor do coeficiente $\mathrm{Dm}$ foi calculado para duas condições distintas: para $\Delta \mathrm{T} / \Delta \mathrm{X} \neq 0$ e para $\Delta \mathrm{T} / \Delta \mathrm{X}=0$.

As amostras de $2,5 \mathrm{~cm}$ de comprimento foram divididas em três partes para a determinação da umidade, ao passo que as de $5,0 \mathrm{~cm}$ de comprimento foram divididas em cinco partes.

$\mathrm{O}$ valor do fluxo de massa, $\mathrm{q}_{\mathrm{m}}(\mathrm{m} / \mathrm{s})\left(\mathrm{g}_{\mathrm{H} 2 \mathrm{O}} / \mathrm{g}_{\mathrm{mtotal}}\right)$ foi calculado multiplicando-se a quantidade de água consumida pela amostra por unidade de tempo pela área da amostra.
Os valores dos coeficientes $D_{T}$ e $D_{M}$ calculados para as diferentes condições de diâmetro, comprimento e granulometria são apresentados nas Tabelas 3 e 4.

TABELA 3. Valores de $D_{T}$ e $D_{M}$ obtidos para as amostras de $2,5 \mathrm{~cm}$ de comprimento para diferentes condições de diâmetro e granulometria.

\begin{tabular}{cccccccc}
\hline $\begin{array}{c}\text { Diâmetro } \\
(\mathrm{cm})\end{array}$ & Peneira & $\mathrm{qm}(\mathrm{m} / \mathrm{s})$ & $\begin{array}{c}\mathrm{D}_{\mathrm{T}} \\
\left(\mathrm{m}^{2} / \mathrm{s} \mathrm{K}\right)\end{array}$ & $\mathrm{D}_{\mathrm{M}}\left(\mathrm{m}^{2} / \mathrm{s}\right)$ & $\begin{array}{c}\mathrm{D}_{\mathrm{M}} \\
\Delta \mathrm{T} / \Delta \mathrm{X}=0\end{array}$ & $\mathrm{DP}\left(\mathrm{m}^{2} / \mathrm{s}\right)$ & $\Delta \mathrm{D}_{\mathrm{M}}(\%)$ \\
\hline 1 & $\# 28$ & $3.39 \mathrm{E}-7$ & $2.04 \mathrm{E}-9$ & $1.87 \mathrm{E}-10$ & $1.51 \mathrm{E}-10$ & $5.99 \mathrm{E}-11$ & 15.53 \\
& $\# 48$ & $3.18 \mathrm{E}-7$ & $1.13 \mathrm{E}-9$ & $1.61 \mathrm{E}-10$ & $1.45 \mathrm{E}-10$ & $4.97 \mathrm{E}-11$ & 7.39 \\
& $\# 100$ & $4.32 \mathrm{E}-7$ & $9.16 \mathrm{E}-9$ & $1.87 \mathrm{E}-10$ & $1.66 \mathrm{E}-10$ & $3.71 \mathrm{E}-11$ & 8.41 \\
2 & $\# 28$ & $3.25 \mathrm{E}-7$ & $3.96 \mathrm{E}-9$ & $2.16 \mathrm{E}-10$ & $2.27 \mathrm{E}-10$ & $1.84 \mathrm{E}-10$ & 28.78 \\
& $\# 48$ & $4.71 \mathrm{E}-7$ & $5.07 \mathrm{E}-9$ & $3.64 \mathrm{E}-10$ & $4.68 \mathrm{E}-10$ & $1.84 \mathrm{E}-10$ & 7.47 \\
& $\# 100$ & $3.93 \mathrm{E}-7$ & $1.73 \mathrm{E}-9$ & $2.00 \mathrm{E}-10$ & $1.82 \mathrm{E}-10$ & $3.86 \mathrm{E}-11$ & 6.66 \\
3 & $\# 28$ & $3.86 \mathrm{E}-7$ & $2.38 \mathrm{E}-9$ & $1.89 \mathrm{E}-10$ & $1.84 \mathrm{E}-10$ & $8.38 \mathrm{E}-11$ & 1.90 \\
& $\# 48$ & $4.81 \mathrm{E}-7$ & $3.24 \mathrm{E}-9$ & $3.21 \mathrm{E}-10$ & $3.38 \mathrm{E}-10$ & $1.87 \mathrm{E}-10$ & 3.65 \\
& $\# 100$ & $5.60 \mathrm{E}-7$ & $5.23 \mathrm{E}-9$ & $4.48 \mathrm{E}-10$ & $4.19 \mathrm{E}-10$ & $2.21 \mathrm{E}-10$ & 4.73 \\
\hline
\end{tabular}

Legenda: $\mathrm{DP}=$ desvio padrão; $\Delta \mathrm{D}_{\mathrm{M}}=$ diferença percentual entre os valores de $\mathrm{D}_{\mathrm{M}}$ calculados considerando-se $\Delta \mathrm{T} / \Delta \mathrm{X} \neq 0$ e $\Delta \mathrm{T} / \Delta \mathrm{X}=0$

TABELA 4. Valores de $\mathrm{D}_{\mathrm{T}}$ e $\mathrm{D}_{\mathrm{M}}$ obtidos para as amostras de $5,0 \mathrm{~cm}$ de comprimento para diferentes condições de diâmetro e granulometria.

\begin{tabular}{|c|c|c|c|c|c|c|c|c|c|}
\hline $\begin{array}{c}\mathrm{D} \\
(\mathrm{cm})\end{array}$ & $\mathrm{P}$ & $\begin{array}{c}\mathrm{qm} \\
(\mathrm{m} / \mathrm{s})\end{array}$ & $\begin{array}{c}\mathrm{D}_{\mathrm{T}} \\
\mathrm{m}^{2} / \mathrm{s} \mathrm{K}\end{array}$ & $\begin{array}{l}\text { DP da } D_{T} \\
\left(\mathrm{~m}^{2} / \mathrm{s} \mathrm{K}\right)\end{array}$ & $\mathrm{D}_{\mathrm{M}}\left(\mathrm{m}^{2} / \mathrm{s}\right)$ & $\begin{array}{c}\text { DP da } D_{M} \\
\left(\mathrm{~m}^{2} / \mathrm{s}\right)\end{array}$ & $\begin{array}{c}\mathrm{D}_{\mathrm{M}} \\
\Delta \mathrm{T} / \Delta \mathrm{X}=0\end{array}$ & $\begin{array}{c}\mathrm{DP} \text { da } \mathrm{D}_{\mathrm{M}} \\
\text { com } \\
\Delta \mathrm{T} / \Delta \mathrm{X}=0 \\
\left(\mathrm{~m}^{2} / \mathrm{s}\right)\end{array}$ & $\begin{array}{l}\Delta \mathrm{D}_{\mathrm{M}} \\
(\%)\end{array}$ \\
\hline \multirow[t]{3}{*}{1} & $\# 28$ & $3.93 \mathrm{E}-7$ & $-1.03 \mathrm{E}-8$ & $1.35 \mathrm{E}-9$ & $2.02 \mathrm{E}-10$ & $2.20 \mathrm{E}-11$ & $5.04 \mathrm{E}-10$ & $3.71 \mathrm{E}-10$ & 60.61 \\
\hline & \#48 & $3.44 \mathrm{E}-7$ & $-2.61 \mathrm{E}-9$ & $6.65 \mathrm{E}-9$ & $2.05 \mathrm{E}-10$ & $2.05 \mathrm{E}-10$ & $2.95 \mathrm{E}-10$ & $1.73 \mathrm{E}-10$ & 30.97 \\
\hline & $\# 100$ & $4.8 \mathrm{E}-7$ & $-5.45 \mathrm{E}-9$ & $6.38 \mathrm{E}-9$ & $2.76 \mathrm{E}-10$ & $1.33 \mathrm{E}-10$ & $5.74 \mathrm{E}-10$ & $3.83 \mathrm{E}-10$ & 49.80 \\
\hline \multirow[t]{3}{*}{2} & \#28 & $3.54 \mathrm{E}-7$ & $-8.6 \mathrm{E}-9$ & $2.01 \mathrm{E}-8$ & $4.33 \mathrm{E}-10$ & $6.8 \mathrm{E}-10$ & $4.46 \mathrm{E}-10$ & $9.67 \mathrm{E}-10$ & 2.09 \\
\hline & \#48 & $4.78 \mathrm{E}-7$ & $4.19 \mathrm{E}-9$ & $7.21 \mathrm{E}-9$ & $3.64 \mathrm{E}-10$ & $3.55 \mathrm{E}-10$ & $3.42 \mathrm{E}-10$ & $2.53 \mathrm{E}-10$ & 21.41 \\
\hline & $\# 100$ & $3.86 \mathrm{E}-7$ & $1.72 \mathrm{E}-9$ & $2.97 \mathrm{E}-7$ & $4.07 \mathrm{E}-10$ & $6.98 \mathrm{E}-9$ & $5.16 \mathrm{E}-10$ & $3.00 \mathrm{E}-10$ & 32.51 \\
\hline \multirow[t]{3}{*}{3} & \#28 & $3.94 \mathrm{E}-7$ & $5.09 \mathrm{E}-9$ & $7.35 \mathrm{E}-9$ & $4.52 \mathrm{E}-10$ & $2.57 \mathrm{E}-10$ & $3.43 \mathrm{E}-10$ & $2.22 \mathrm{E}-10$ & 19.39 \\
\hline & \#48 & $4.43 \mathrm{E}-7$ & $5.06 \mathrm{E}-9$ & $9.88 \mathrm{E}-9$ & $4.14 \mathrm{E}-10$ & $1.25 \mathrm{E}-10$ & $2.96 \mathrm{E}-10$ & $9.22 \mathrm{E}-11$ & 23.50 \\
\hline & $\# 100$ & $4.50 \mathrm{E}-7$ & $-1.28 \mathrm{E}-8$ & $3.18 \mathrm{E}-9$ & $3.85 \mathrm{E}-10$ & $7.28 \mathrm{E}-10$ & $5.70 \mathrm{E}-10$ & $9.03 \mathrm{E}-10$ & 23.59 \\
\hline
\end{tabular}

Observa-se alguns valores negativos para os valores de $\mathrm{D}_{\mathrm{t}}$ e $\mathrm{D}_{\mathrm{m}}$ quando considerado o gradiente de temperatura. Isso se deve ao fato de o gradiente de temperatura ter se apresentado negativo em alguns casos.

As Figuras 8 e 9 mostram a influência da granulometria no cálculo do coeficiente $\mathrm{D}_{\mathrm{M}}$ para $\Delta \mathrm{T} / \Delta \mathrm{X}=0 \mathrm{e}$ $\Delta \mathrm{T} / \Delta \mathrm{X} \neq 0$, respectivamente.

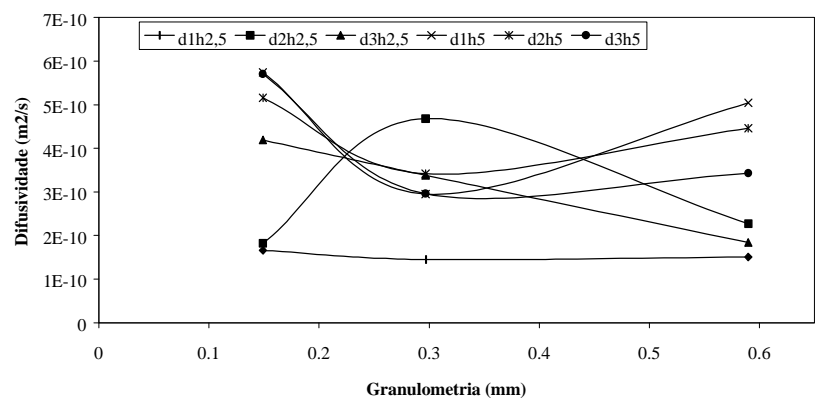

Legenda: $\mathrm{di}=$ amostras com diâmetro $\mathrm{i}(\mathrm{i}=1,2,3) . \mathrm{hj}=$ amostras com comprimento $\mathrm{j}$ $(\mathrm{j}=2,5$ ou 5$)$

FIGURA 8. Influência da granulometria na determinação de $\mathrm{D}_{\mathrm{M}}$ considerando-se o gradiente de temperatura desprezivel 


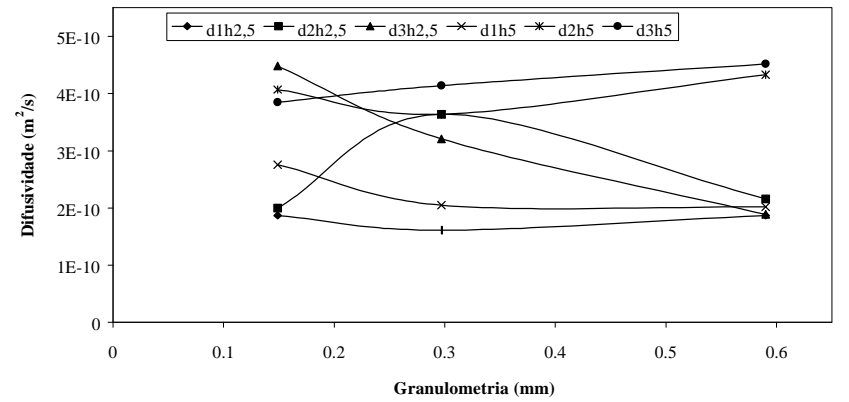

Legenda: $d i=$ amostras com diâmetro $i(i=1,2,3) . h j=$ amostras com comprimento $j$ $(\mathrm{j}=2,5$ ou 5$)$

FIGURA 9. Influência da granulometria na determinação de $\mathrm{D}_{\mathrm{M}}$ considerando-se o gradiente de temperatura

Analisando-se a Figura 8 percebe-se que, para as amostras de 5,0cm de comprimento, as curvas apresentam a mesma tendência, onde a difusividade máxima corresponde à menor granulometria (0,149mm).

A Figura 9 mostra que (exceto para as amostras $\mathrm{d} 2 \mathrm{~h} 2,5$ e d3h2,5) a difusividade tende a ser constante com a variação da granulometria, quando se considera o gradiente de temperatura no cálculo de $\mathrm{D}_{\mathrm{m}}$. Portanto, na avaliação do coeficiente de transferência de massa, para o experimento de coluna aberta, a contribuição do gradiente de temperatura deve ser considerada.

As Figuras 10 e 11 mostram a influência do comprimento do corpo-de-prova na determinação de $\mathrm{D}_{\mathrm{m}}$ para $\Delta \mathrm{T} / \Delta \mathrm{X}=0$ e $\Delta \mathrm{T} / \Delta \mathrm{X} \neq 0$, respectivamente.

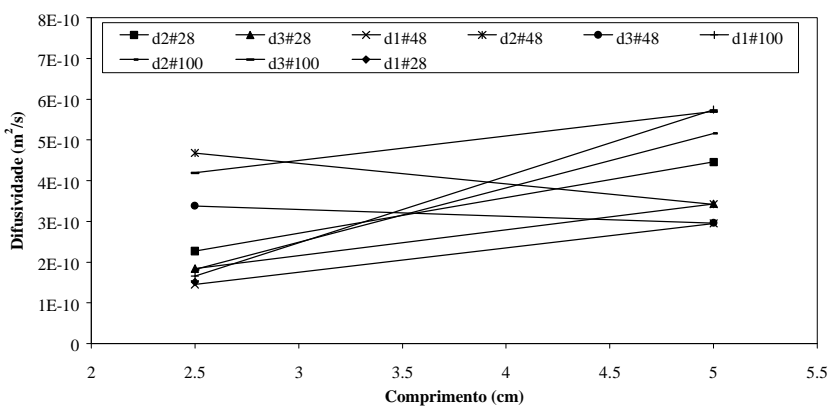

FIGURA 10. Influência do comprimento na determinação de $\mathrm{D}_{\mathrm{M}}$ considerando-se o gradiente de temperatura desprezivel

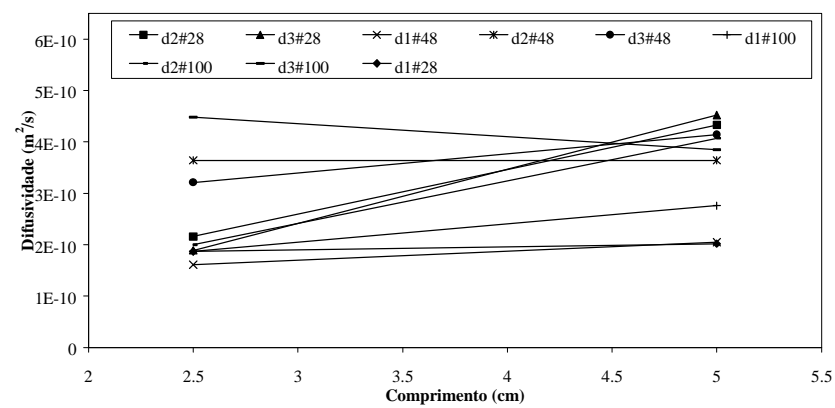

FIGURA 11. Influência do comprimento na determinação de $\mathrm{D}_{\mathrm{M}}$ considerando-se o gradiente de temperatura.
Analisando-se a Figura 10, percebe-se que existe uma tendência de a difusividade aumentar com o aumento do comprimento. Apenas a amostra de diâmetro $2,0 \mathrm{~cm}$ e peneira 48 (d2-\#48) apresentou uma tendência diferente.

A Figura 11 mostra que a tendência do aumento da difusividade com o aumento do comprimento da amostra diminui quando se leva em consideração o gradiente de temperatura no cálculo. As Figuras 12 e 13 mostram a influência do diâmetro do corpo-de-prova na determinação de $\mathrm{D}_{\mathrm{m}}$ para $\Delta \mathrm{T} / \Delta \mathrm{X}=0$ e $\Delta \mathrm{T} / \Delta \mathrm{X} \neq 0$, respectivamente.

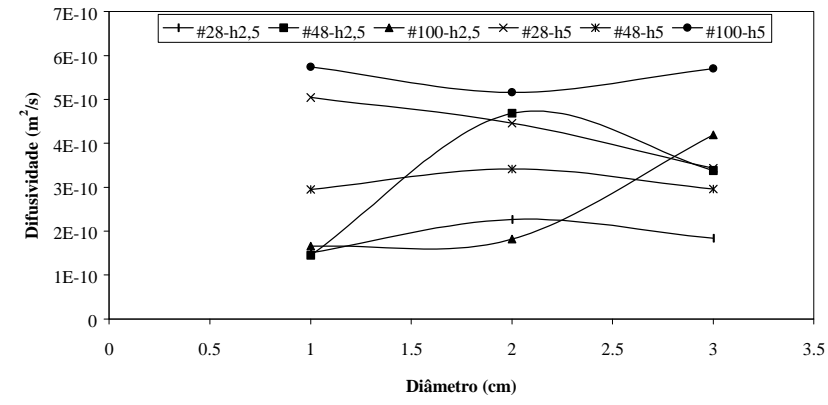

FIGURA 12. Influência do diâmetro do corpo-de-prova na determinação de $\mathrm{D}_{\mathrm{M}}$ desconsiderando-se o gradiente de temperatura

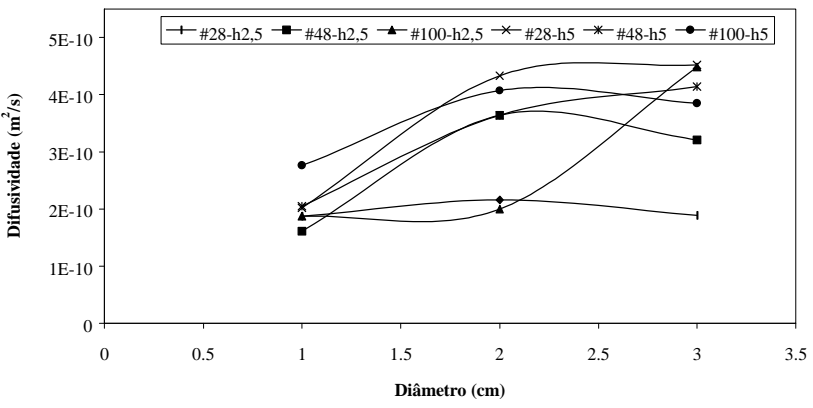

FIGURA 13. Influência do diâmetro do corpo-de-prova na determinação de $\mathrm{D}_{\mathrm{M}}$ considerando-se o gradiente de temperatura

Analisando-se as Figuras 12 e 13, percebe-se que a difusividade é influenciada pelo diâmetro do corpo-de-prova, considerando-se ou não o gradiente de temperatura.

Os resultados dos experimentos de coluna aberta mostram que a determinação do coeficiente de transferência de massa é influenciado pelo gradiente de temperatura, pelo comprimento, pelo diâmetro e pela granulometria.

\section{5 - CONCLUSÕES}

- Nos experimentos com o equipamento de coluna fechada, a maior influência na avaliação da condutividade térmica foi devida aos valores de fluxo de calor obtidos. Os resultados dos experimentos conduzidos nesse equipamento mostram que a migração de umidade deve ser considerada. 
- O comprimento e a granulometria têm influência na determinação da condutividade térmica, ao passo que o diâmetro não interfere no valor da mesma.

- Para os experimentos conduzidos no equipamento de coluna aberta o gradiente de temperatura deve ser considerado na determinação da difusividade mássica. A difusividade mássica é influenciada pelo comprimento e pelo diâmetro da amostra.

- A granulometria interfere na determinação da condutividade térmica, assim como na difusividade mássica.

\section{6 - REFERÊNCIAS BIBLIOGRÁFICAS}

[1] LUIKOV, A. V.; MIKHAILOV, YU, A. 1965. Theory of Energy and Mass Transfer. Pergamon Press. Oxford. 392p.

[2] LUIKOV, A. V. System of differential equations of heat and mass transfer in capillary porous bodies. Int. J. Heat and Mass Transfer, v. 18, p. 1- 14, 1973.

[3] PHILIP, J. R.; DE VRIES, D. A. Moisture movement in porous materials under temperature gradients. Trans. Amer. Geophys. Unin., v. 38, p. 222-232, 1957.

[4] SHIKI, M. P, PARK, K. J. Determination of Simultaneous Heat and Mass Transfer Coefficients for Food Products. Drying 98: Proceedings of the $11^{\text {th }}$ International Drying Symposium. Thessaloniki-Greece: Ziti Editions, v. B, p. 1288-1294, 1998.

[5] TREYBAL, R. E. 1968. Mass Transfer Operation. McGrawHill Book Co., New York, NY. 695p. 